\title{
The evolution of spondylarthritis - a therapeutic challenge
}

\author{
Cristina Radu', Maria Laura Groseanu ${ }^{1,2}$, Ruxandra Ionescu ${ }^{1,2}$ \\ ${ }^{1}$ Department of Internal Medicine and Rheumatology, "Sf. Maria" Clinical Hospital, \\ Bucharest, Romania \\ 2"Carol Davila" University of Medicine and Pharmacy, Bucharest, Romania
}

ABSTRACT
We are presenting the case of a 36 years old male diagnosed at the age of 20 with reactive arthritis and at 27
years with ankylosing spondylitis. During the course of the disease, the patient followed treatment with non-
steroidal anti-inflammatory drugs, Sulfasalazine and biological therapy. The first two anti-TNF had a very good
response. During Adalimumab therapy the patient conceived a child without any teratogenic effect on it. The effect
of the two anti-TNF has been long-lasting, but the effectiveness has decreased on the other two. The future of this
patient lies in blocking a new molecule in ankylosing spondylitis named IL-17, so we are expecting the disease
response to the new biological introduced into ankylosing spondylitis, named Secukinumab.
Keywords: spondylarthritis, anti-TNF, Secukinumab

\section{INTRODUCTION}

Ankylosing spondylitis (AS) is a chronic, multisystem inflammatory disorder primarily involving the sacroiliac (SI) joints and the axial skeleton, including also peripheral arthritis, enthesitis, and extra-articular organ involvement (1-4). The age of onset of AS is usually from the late teens to age 40 years (5). Prevalence rates of AS are approximately equal in men and women. However, men have more severe radiographic changes in the spine and hips than women (6).

Long-term treatment goals are to maximise quality of life through control of signs and symptoms, prevention of structural damage and preservation of physical function (7).

The introduction of tumour necrosis factor (TNF)-blocking therapy has revolutionized the management of ankylosing spondylitis (AS) over the last decade. However, up to $40 \%$ of patients do not respond to or cannot tolerate TNF inhibitors, (8) and loss of efficacy can occur over time (9). Not many studies were performed in case of failure of more than two anti-TNF, but it seems that the lack of two anti-TNF is predictive of the ineffectiveness of the third anti-TNF.
New therapeutic targets are expected in our patient's case to improve the signs and symptoms of the disease improving health-related quality of life.

\section{CASE PRESENTATION}

We are presenting the case of a 36 years old male with disease onset at the age of 17 with bilateral metatarsophalangeal II arthritis, interpreted as gout. At the age of 20, after a diarrheal episode, presents left knee arthritis and bilateral metatarsalphalangeal II arthritis. Biologically presents inflammatory syndrome, anti-Yersinia antibodies + and slight condensation of the left iliac joints versus right at basin radiography. The diagnosis was reactive arthritis; antibiotics, non-steroidal anti-inflammatory and Sulfasalazine up to $2 \mathrm{~g}$ /day were recommended, discontinued by the patient after 2 months.

In 2007, the patient presented to the St. Mary's Clinical Hospital with chronic inflammatory back pain, bilateral knee arthritis, inflammatory syndrome and negative HLA B27. The diagnosis was peripheral spondylarthritis and the treatment given was with Sulfasalazine up to $2.5 \mathrm{~g} /$ day, but the 
treatment was stopped by the patient after 2 months.

Between 2008 and 2009 at "Dr. Carol Davila" Central Military Emergency the patient is treated with Remicade, stopped due to loss of response (medical data not available).

In May 2013, the patient presented in our clinic with severe inflammatory cervical and lumbar pain, arthralgias of the elbows, knees, ankles and metatarsophalangeal. He had already developed modified mobility indices, anterior projection of the cephalic extremity with thoracic kyphosis, ankylosis in elbow flexion, ankle semiankylosis. The disease was very active: ESR $70 \mathrm{~mm} / \mathrm{h}, \mathrm{CRP}$ $110.7 \mathrm{mg} / 1$, BASDAI $=8.75$, with chronic sac-

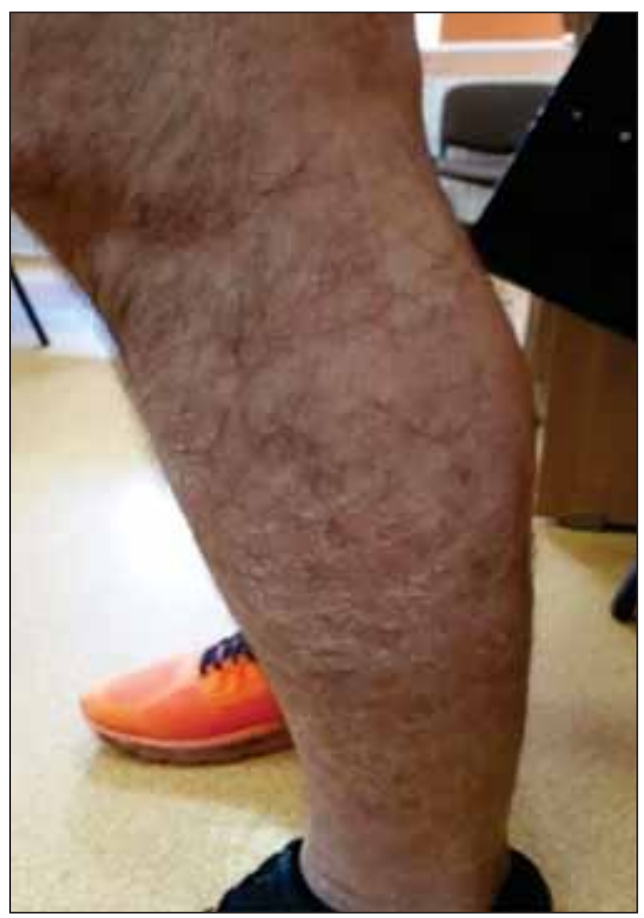

FIGURE 1. Psoriatic lesions

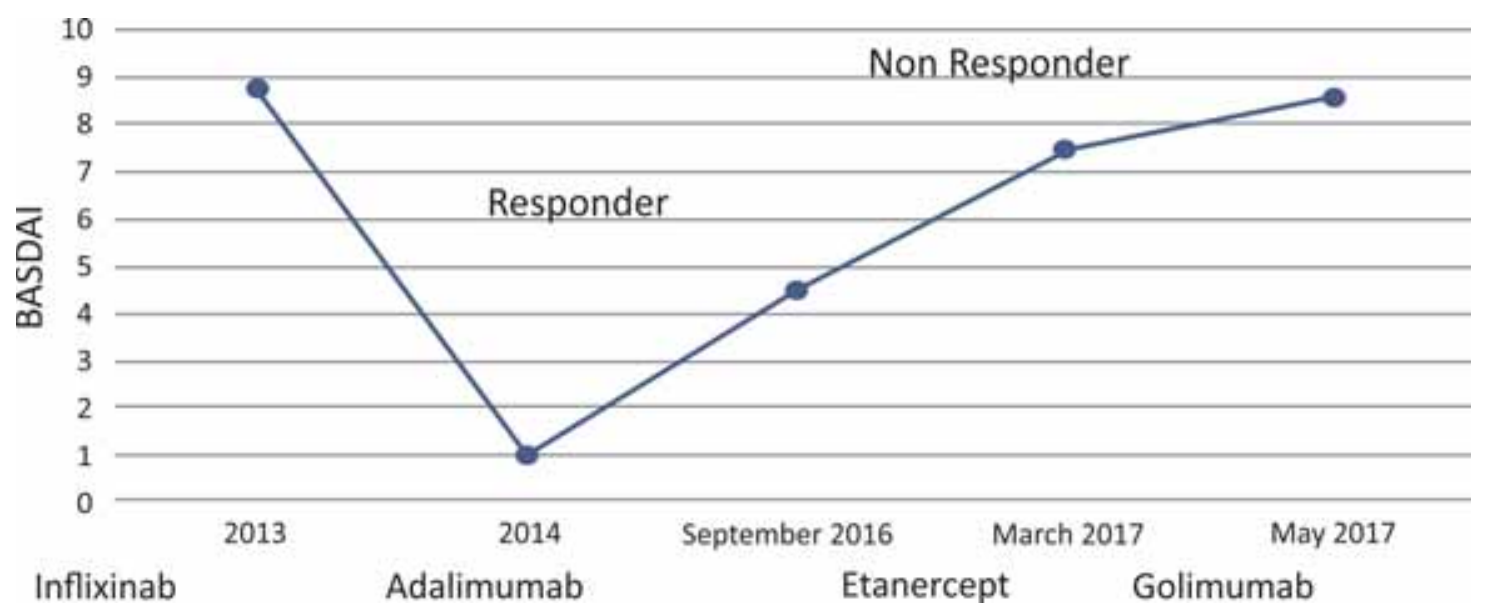

FIGURE 2. The evolution of BASDAI during the biological treatment: It is noticeable that during the first two anti-TNF, the patient lost inefficiency after longer time, while in the case of the other two efficacy decreased much faster roilitis (left $>$ right) and acute (right $>$ left) on MRI, so we decided to start Adalimumab. After 3 months he had no clinical symptoms, no inflammatory syndrome and BASDAI $=1$. The disease was in remission until March-April 2016 when, despite maintaining the clinical benefit of anti-TNF, a slight increase in $\mathrm{C}$ reactive protein was observed, with the occurrence of a psoriasiform exanthema in the calves. Anti-Humira antibodies were negative and acid-serum drug level was at the lower limit of normal. The patient announces that he is the father of a four months healthy baby.

In September 2016 the patient had high disease activity $(\mathrm{BASDAI}=4.5$, BASDAI-CRP $=3.5)$, inflammatory syndrome and aggravation of psoriasis lesions. He was switched on Enbrel with insufficient response at the 6-month evaluation and the spread of psoriasis lesions (Fig. 1). The patient was switched on Golimumab. A psoriasiform eruption biopsy was taken by the dermatologist, the diagnosis was anti-TNF-induced psoriasis. The patient performed 10 UVB rays with the disappearance of psoriasis lesions.

At the 3-month assessment (May 2017), disease activity persists (BASDAI $=8.6$, ASDAS$\mathrm{CRP}=6.44$ ) with a major inflammatory syndrome $(\mathrm{ESR}=89 \mathrm{~mm} / \mathrm{h}, \mathrm{CRP}=273.78 \mathrm{mg} / \mathrm{L})$. The patient doesn't show signs of infection or other changes in laboratory tests including negative hepatitis and Quantiferon. 
Due to the fact that the patient is a failure on four anti-TNF, we decided to change the biological class to Secukinumab (Fig. 2).

\section{DISCUSSIONS}

\section{Anti-TNF-Alpha-Induced Psoriasis - An Unusual Paradox}

Our patient developed a psoriasiform eruption during Adalimumab treatment, worsened during Etanercept treatment. The question that raised up was if the patient had psoriatic arthritis with no previous eruption or if the eruption was a dermatological side effect of anti-TNF-alpha treatment? However, several aspects of this phenomenon provide evidence for the idea that it is a side effect of anti-TNF- $\alpha$ agents: absence of a personal or family history of psoriasis, the temporal relationship between anti-TNF- $\alpha$ treatment and the appearance of cutaneous lesions, and the clinical improvement observed after discontinuation of therapy.

Since an initial report in 2003 (10) numerous cases of typical psoriasis and psoriasiform lesions induced by anti-TNF-alpha therapy have been described, and two systematic literature reviews have been performed, the most recent analysing 207 cases (11). This paradoxical side effect has been described in nearly all conditions treated with anti-TNF and with all anti-TNF agents $(12,13)$. The time between anti-TNF- $\alpha$ administration and development of psoriatic lesions varies widely (13) suggesting that an environmental trigger may also be involved. The reported cutaneous lesions are plaque, pustular $(50 \%)$ or guttate-type psoriasis. The most frequently affected areas are the scalp and flexures and palmoplantar areas (14). The incidence rate of psoriasis in an rheumatic diseases population from the UK was estimated at 1.04/1,000 person-years (15). Data from the Spanish biological register found similar results, with a global incident rate of 2.31/1,000 patient-years (16).

The mechanism underlying the induction or exacerbation of psoriatic lesions by anti-TNF- $\alpha$ antagonists is not clear (17). The most popular is a disequilibrium in cytokine balance. TNF- $\alpha$ suppresses the development of plasmacytoid dendritic cells (pDCs), a major cellular component for the production of IFN type I such as IFN- $\alpha$ (17$19)$. IFN- $\alpha$ is an inducer of certain chemokine re- ceptors on T cells (CXCR3) that induce T-cell migration to the skin (17-19). Polymorphisms of genes involved in cytokine production, such as IL-23-R, are also probably involved (17).

Biopsy should be considered in all patients with new-onset psoriatic lesions to eliminate differential diagnosis for psoriasis mimickers. A triggering event including infection, a stressful life or intake of new drugs must be systematically investigated. In our case, the diagnosis was confirmed by skin biopsy, the eruption correlated with a major stress in patients life. According to Collamer's algorithm (14), patients with psoriasis covering $<5 \%$ of body surface area should be treated with topical treatments (corticosteroids, keratolytics and vitamin D analogs); for lesions covering $>5 \%$ of body surface area and palmoplantar disease, topical therapies, occlusive therapy and UV phototherapy should be given. However, in the severe cases, treatment should include both withdrawal of anti-TNF and consequent treatment of psoriasis. In our case, topical steroids, PUVA and switching antiTNF lead to psoriasis resolution.

\section{Fertility and pregnancy safety of antiTNF-alpha medication}

Little is known about anti-TNF use in males with rheumatic diseases that want to conceive. Several studies have sought to determine the impact of anti-TNF $\alpha$ medications on sperm viability (20-22). The most recent study confirms that sperm quality in patients with active AS and after receiving short- and long-term TNF- $\alpha$ blocker therapy is comparable to sperm quality in healthy controls (20). A total of 17 pregnancies conceived by men taking Infliximab have been reported (21). One pregnancy resulted in a first trimester miscarriage in a woman with Addison's disease and a prior miscarriage. The remaining pregnancies resulted in healthy babies. These reports suggest that the anti-TNF $\alpha$ medications promoted male fertility with no harm for the babies. According to recent EULAR recommendations, continuation of TNF inhibitors in female patients during the first part of pregnancy should be considered (23). Etanercept and Certolizumab may be considered for use throughout pregnancy due to low rate of transplacental passage (23). Infliximab and Adalimumab may preferentially be stopped at 20 weeks and Etanercept at week 30-32 of pregnancy. Sound 
evidence for fetal/child safety is still lacking for biologics approved less $<5$ years ago (23). Current evidence for Adalimumab indicates no increased risk for congenital malformation and can be continued up to gestational week 20 , if indicated can be used through pregnancy and is compatible with breastfeeding (23).

\section{TNF non-responders - a sad but hopeful story}

Around $10-30 \%$ of patients do not respond to initial anti-TNF treatment and $23-46 \%$ of patients lose response over time $(24,25)$. A common mechanism for the loss of response is immunogenicity due to the formation of antibodies against the $\mathrm{TNF} \alpha$ antagonists. Primary nonresponse refers to patients who do not respond adequately to the initial loading doses of a biologic agent $(25,26)$. These patients are found to have adequate drug levels and no antibodies. They may not respond to the particular mechanism of action of the drug, and switching to a medication in a different class is recommended $(25,26)$, Secondary loss of response refers to patients who had previously responded to a biologic agent. The drug dosing should be increased if drug levels are low and antibodies are not present, switched to another drug in the class if drug levels are low and antibody levels are high, or switched to another drug mechanism if drug levels are high and antibodies are not present $(25,26)$. Observational studies have demonstrated ADA levels $>4.5 \mathrm{mcg} / \mathrm{ml}$ are associated with an increased likelihood of maintaining response $(27,28)$. For our patient Adalimumab serum level was $7.5 \mathrm{mcg} / \mathrm{ml}$, although the cutoff for was $10 \mathrm{mcg} / \mathrm{ml}$. No anti-Adalimumab antibodies were present but the drug level was low suggesting that antibodies might be present but under the limit of detection.

Yanai et al. (26) have shown that patients with no/low-titer ADA responded significantly better to dose intensification (increase in the dose or dose frequency) compared with the anti-TNF switch. Still, this strategy is not approved for patients with AS, so our option at that moment was several anti-TNF switches. Several studies have confirmed the efficacy of switching to a second or third TNFi although overall effectiveness seems to be somewhat lower than in non-switchers (29, 30). No data are available regarding the third and fourth anti-TNF switch.

There is significant unmet need in patients with ankylosing spondylitis who have inadequate response or intolerance to anti-tumour necrosis factor treatment. Secukinumab, an anti-interleukin17A monoclonal antibody, significantly improved signs and symptoms of AS in the MEASURE 1 and MEASURE 2 study (31). Around 30\% patients were not anti-TNF naive. Up to $60 \%$ of antiTNF inadequate responders had achieved ASAS20 response by 52 weeks (31). Recently, longer term 2-year data were available, demonstrating that Secukinumab provided sustained improvements in signs and symptoms of AS, with improved physical function regardless of anti-TNF status (31).

\section{CONCLUSIONS}

AntiTNF- $\alpha$ therapies are effective for induction and maintenance of remission in patients with ankylosing spondylitis, allowing young male patients to have a normal social and family life. However, clinicians face many challenges in determining the best course of action when a patient loses response or develops side effects. When patients are found to have continued active inflammation despite having undergone biologic therapy, the first determination should be whether this represents a primary nonresponse to the drug's mechanism of action or a secondary loss of response due to inadequate drug levels and/or antibody formation to the drug. In this cases, new treatment options like anti IL17 antibodies have just became available.

Conflict of interest: none declared Financial support: none declared

\section{REFERENCES}

1. Cawley M.I., Chalmers T.M., Ball J. Destructive lesions of vertebral bodies in ankylosing spondylitis. Ann Rheum Dis. 1971 Sep. 30(5):539-40.

2. Hanson J.A., Mirza S. Predisposition for spinal fracture in ankylosing spondylitis. AJR Am J Roentgenol. 2000 Jan. 174(1):150.

3. Hunter T. The spinal complications of ankylosing spondylitis. Semin Arthritis Rheum. 1989 Dec. 19(3):172-82.

4. Sutherland R.I., Matheson D. Inflammatory involvement of vertebrae in ankylosing spondylitis. J Rheumatol. 1975 Sep. 2(3):296-302.

5. Braun J., Sieper J. Ankylosing spondylitis. Lancet. 2007 Apr 21. 369(9570):1379-90. 
6. Lee W., Reveille J.D., Davis J.C. Jr. et al. Are there gender differences in severity of ankylosing spondylitis? Results from the PSOAS cohort. Ann Rheum Dis. 2007 May. 66(5):633-8.

7. Braun J., van den Berg R., Baraliakos X. et al. 2010 update of the ASAS/EULAR recommendations for the management of ankylosing spondylitis. Ann Rheum Dis 2011; 70:896-904.

8. Braun J., Kiltz U., Heldmann F. et al. Emerging drugs for the treatment of axial and peripheral spondyloarthritis. Expert Opin Emerg Drugs 2015; 20:1-14.

9. Baraliakos X., Listing J., Fritz C. et al. Persistent clinical efficacy and safety of infliximab in ankylosing spondylitis after 8 years-early clinical response predicts long-term outcome. Rheumatology (Oxford) 2011; 50:1690-9.

10. Viguier M., Richette P., Bachelez H. et al. Paradoxical adverse effects of anti-TNF-alpha treatment: onset or exacerbation of cutaneous disorders. Expert Rev Clin Immunol 2009; 5:421-31.

11. Collamer A.N., Battafarano D.F. Psoriatic skin lesions induced by tumor necrosis factor antagonist therapy: clinical features and possible immunopathogenesis. Semin Arthritis Rheum 2010; 40:233-40.

12. Toussirot E., Aubin F. Paradoxical reactions under TNF- $\alpha$ blocking agents and other biological agents given for chronic immunemediated diseases: an analytical and comprehensive overview RMD Open. 2016; 2(2): e000239. Published online 2016 Jul 15.

13. Mocci G., Marzo M., Papa A., Armuzzi A., Guidi L. Dermatological adverse reactions during anti-TNF treatments: Focus on inflammatory bowel disease Journal of Crohn's and Colitis 2013; 7(10); 769-779

14. Collamer A.N., Guerrero K.T., Henning J.S. et al. Psoriatic skin lesions induced by tumor necrosis factor antagonist therapy: a literature review and potential mechanisms of action. Arthritis Rheum 2008; 59:996-1001.

15. Harrison M.J., Dixon W.G., Watson K.D. et al. Rates of newonset psoriasis in patients with rheumatoid arthritis receiving anti-tumour necrosis factor alpha therapy: results from the British Society for Rheumatology Biologics Register. Ann Rheum Dis 2009; 68:209-15.

16. Hernandez M.V., Sanmarti R., Canete J.D. et al. Cutaneous adverse events during treatment of chronic inflammatory rheumatic conditions with tumor necrosis factor antagonists: study using the Spanish registry of adverse events of biological therapies in rheumatic diseases. Arthritis Care Res 2013; 65:2024-31.

17. Collamer A.N., Battafarano D.F. Psoriatic skin lesions induced by tumor necrosis factor antagonist therapy: clinical features and possible immunopathogenesis. Semin Arthritis Rheum 2010; 40:232-40.

18. Palucka A.K., Blanck J.P., Bennett L. et al. Cross-regulation of TNF and IFN-alpha in autoimmune diseases. Proc Natl Acad Sci USA 2005; 102:3372-7.

19. Seneschal J., Milpied B., Vergier B. et al. Cytokine imbalance with increased production of interferon-alpha in psoriasiform eruptions associated with antitumour necrosis factor-alpha treatments. Br J Dermatol 2009; 161:1081-8.

20. Wildi L.M., Haraoui B. Reversible male infertility under treatment with an anti-TNF-a agent, a case report, Ann Rheum Dis, 2012, vol. 71 (pg. 473-4)

21. Puchner R., Danninger K., Puchner P., Pieringer H. Impact of TNF-blocking agents on male sperm characteristics and pregnancy outcomes in fathers exposed to TNF-blocking agents at time of conception, Clin Exp Rheumatol, 2012, vol. 30 (pg. 765-7)

22. Micu M.C., Micu R., Surd S., Gîrlovanu M., Bolboacă S.D., Ostensen M. TNF- $\alpha$ inhibitors do not impair sperm quality in males with ankylosing spondylitis after short-term or long-term treatment. Rheumatology (Oxford). 2014 Jul; 53(7):1250-5.

23. Götestam Skorpen C., Hoeltzenbein M., Tincani A. et al. The EULAR points to consider for use of antirheumatic drugs before pregnancy, and during pregnancy and lactation. Ann Rheum Dis Published Online First: February 17, 2016 as 10.1136/ annrheumdis-2015-208840

24. Coates L., Marzo-Ortega H., Bennet A., Emery P. Anti-TNF Therapy in Ankylosing Spondylitis: Insights for the ClinicianTher Adv Musculoskelet Dis. 2010 Feb; 2(1): 37-43.

25. Roda G. Jharap B., Neeraj N., Frederic J.C. Loss of Response to Anti-TNFs: Definition, Epidemiology, and Management Clin Transl Gastroenterol. 2016; 7(1): e135. Published online 2016 Jan 7.

26. Yanai H., Lichtenstein L., Assa A. et al. Levels of drug and antidrug antibodies are associated with outcome of interventions after loss of response to infliximab or adalimumab. Clin Gastroenterol Hepatol 2015; 13: 522-530.

27. Roblin X., Rinaudo M., Del Tedesco E. et al. Development of an algorithm incorporating pharmacokinetics of adalimumab in inflammatory bowel diseases. Am J Gastroenterol 2014; 109 : 1250-1256.

28. De Vries M.K., Brouwer E., Van der Horst-Bruinsma I.E., Spoorenberg A., Van Denderen J.C., Jamnitski A., Nurmohamed M.T., Dijkmans B.A., Aarden L.A., Wolbink G.J. Decreased clinical response to adalimumab in ankylosing spondylitis is associated with antibody formation. Ann Rheum Dis. 2009; 15:1787-1788. doi: 10.1136/ard.2009.109702.

29. Paccou J., Solau-Gervais G., Houvenagel E., Salleron J., Luraschi H., Philippe P. et al. Efficacy in current practice of switching between anti-tumour necrosis factor- $\alpha$ agents in spondyloarthropathies Rheumatology (Oxford) (2011) 50 (4): 714 720.

30. Lie E., Van der H.D., Uhlig T., Mikkelsen K., Rodevand E., Koldingsnes W., Kaufmann C., Kvien T.K. Effectiveness of switching between TNF inhibitors in ankylosing spondylitis: data from the NOR-DMARD register. Ann Rheum Dis. 2011; 15:157-163.

31. Cheung P. Anti-IL17A in Axial Spondyloarthritis - Where Are We At? Front. Med., 18 January 2017; 4:1. 